\title{
Role of nanocrystalline domain size on the electrochemical double-layer capacitance of high edge density carbon nanostructures
}

\author{
Stephen M. Ubnoske, Department of Mechanical Engineering and Materials Science, Duke University, Durham, North Carolina 27708, USA \\ Akshay S. Raut, Charles B. Parker, and Jeffrey T. Glass, Department of Electrical and Computer Engineering, Duke University, Durham, North \\ Carolina 27708, USA \\ Brian R. Stoner, Research Triangle Institute (RTI) International, Durham, North Carolina 27709, USA \\ Address all correspondence to Stephen M. Ubnoske at stephen.ubnoske@duke.edu
}

(Received 18 December 2014; accepted 10 March 2015)

\begin{abstract}
Nanostructured carbon materials, especially activated carbon, carbon nanotubes, and graphene, have been widely studied for supercapacitor applications. To maximize the efficacy of these materials for electrochemical energy storage, a detailed understanding of the relationship between the nanostructure of these materials and their performance as supercapacitors is required. A fundamental structural parameter obtained from the Raman spectra of these materials, the in-plane correlation length or nanocrystalline domain size, is found to correlate with the electrochemical capacitance, regardless of other morphological features. This correlation for a common nanostructural characteristic is believed to be the first result of its kind to span several distinct nanostructured carbon morphologies, including graphene-carbon nanotubes hybrid materials, and may allow more effective nanoscale engineering of supercapacitor electrode materials.
\end{abstract}

\section{Introduction}

Nanostructured carbon materials have been widely studied for applications in electrochemical energy storage, including activated carbon, ${ }^{[1-3]}$ carbon nanotubes $(\mathrm{CNTs}),{ }^{[4-7]}$ and graphene. ${ }^{[8-11]}$ Applications of such devices include hybrid structures in automotive energy storage, ${ }^{[12]}$ flexible electronics, ${ }^{[13-15]}$ and neural stimulation electrodes. ${ }^{[16]}$ In order to optimize the performance of such devices, a clear understanding of the relationship between the physical properties of nanostructured carbon materials and electrochemical capacitance is required. As a nondestructive process, determination of nanocrystalline domain size using the Raman $I_{\mathrm{D}} / I_{\mathrm{G}}$ ratio, ${ }^{[17-20]}$ referred to interchangeably as graphitic cluster size or in-plane correlation length, ${ }^{[21]}$ provides a facile and inexpensive method to engineer carbon nanostructures for energy storage applications. In this study, we present a relationship between this nanocrystalline domain size and the specific capacitance of various carbon nanostructures grown by plasma-enhanced chemical vapor deposition (PECVD) and measured by Raman spectroscopy. Previous works ${ }^{[22-26]}$ have suggested a relationship between graphitic edge planes and specific capacitance for graphite, glassy carbon, highly ordered pyrolytic graphite, carbon nanofibers, multi-walled carbon nanotubes, and doped graphene. This work is believed to be the first to quantify the relationship between structural defects, largely contributed by few-layered graphene (FLG) edge planes, and the specific capacitance in terms of nanocrystalline domain size for graphene-CNT hybrid materials. It is also believed to be the first study of its kind across materials that span such a large range of domain sizes.

\section{Materials and methods}

The details of the PECVD growth system are described elsewhere, ${ }^{[27]}$ as is the deposition process for growth of the graphenated carbon nanotubes (g-CNTs) used in this study. ${ }^{[28]}$ In brief, nanostructures were grown in a $915 \mathrm{MHz}$ microwave PECVD reactor using $50 \AA \mathrm{Fe}$ catalyst deposited on silicon. Prior to deposition, the substrates were heated to the desired deposition temperature in $100 \mathrm{sccm} \mathrm{NH}$, followed by striking and stabilizing the plasma at 21 Torr and $2.15 \mathrm{~kW}$ magnetron input power. The substrates were pretreated for several minutes in the ammonia plasma to dewet the Fe catalyst film to form nanoparticles. The gas flow was subsequently changed to $150 \mathrm{sccm} \mathrm{CH}_{4}$ and $50 \mathrm{sccm} \mathrm{NH} \mathrm{NH}_{3}$ for the desired deposition time. The graphene and CNT formed simultaneously during the growth process. Possible mechanisms for this formation have been discussed elsewhere. ${ }^{[28,29]}$ In contrast to the hightemperature conditions of g-CNT growth, which results in a high foliate density but poor nanotube alignment, vertically aligned graphenated carbon nanotubes (VA g-CNTs) were achieved by reducing deposition temperature to $900{ }^{\circ} \mathrm{C}$ and extending deposition time to $30 \mathrm{~min}$. Hierarchical structures of few-layered graphene and carbon nanotubes were created by further extending the deposition time, hereafter referred to as carbon nanosheets on carbon nanotubes or CNS-on-CNTs. 


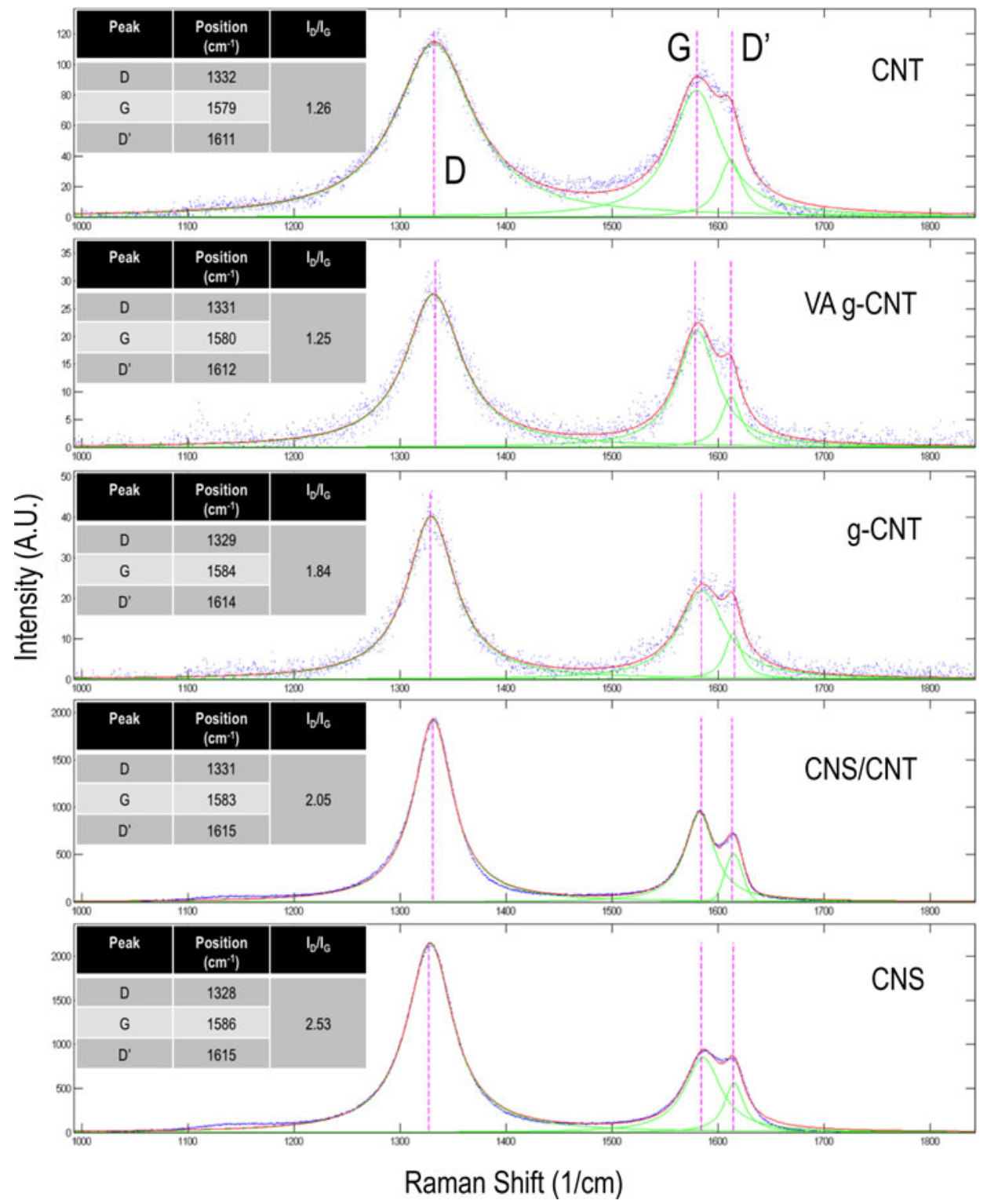

Figure 1. Raman spectra and Lorentzian deconvolution of the first-order Raman-active modes in various carbon nanostructures grown by MPECVD. The peaks shown are the $\mathrm{D}, \mathrm{G}$, and $\mathrm{D}^{\prime}$ in order of increasing wavenumber.

Raman spectroscopy was carried out using a Horiba Jobin Yvon LabRam ARAMIS spectrometer operating with a $633 \mathrm{~nm}$ HeNe laser. The $D$ and $G$ Raman modes were deconvolved as Lorentzian peaks and the $D^{\prime}$ was modeled as a Gaussian peak, as suggested by Ferrari and Robertson ${ }^{[21]}$ and Mennella et al. ${ }^{[30]}$ The ratio of the $D$ and $G$ band intensities were calculated from the intensities of the isolated peaks. The Raman spectra of the nanostructured materials and deconvolution of the peaks are shown in Fig. 1. Scanning electron micrographs of the various nanostructures examined in this study are shown in Fig. 2. Carbon nanostructures were imaged with a FEI XL30 SEM-FEG scanning electron microscope.
The electrochemical cell and sample preparation have been described in detail elsewhere. ${ }^{[31]}$ A three-terminal cell (K0235 by Princeton Applied Research) was used with the nanostructure under study as the working electrode, a Pt mesh as the counter-electrode, and an $\mathrm{Ag}$ wire in $1 \mathrm{M}$ tetrabutylammonium and $0.01 \mathrm{M} \mathrm{AgNO}_{3}$ in acetonitrile as the reference electrode. The electrolyte used was $1 \mathrm{M} \mathrm{LiClO}_{4}$ in acetonitrile. To perform electrochemical measurements, the nanostructured electrode was mounted on a piece of sheet metal using copper tape, and electrical contact was made by painting conductive silver epoxy on the nanostructure side. The nominal active area of the electrode was $1.43 \mathrm{~cm}^{2}$, defined by a PTFE gasket. 


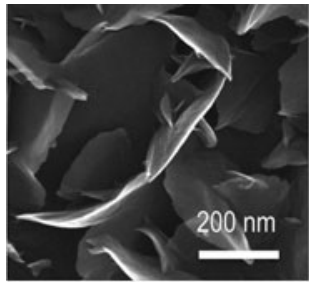

(a)

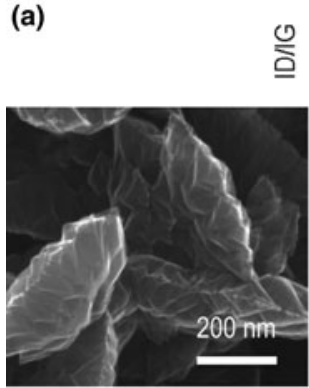

(b)

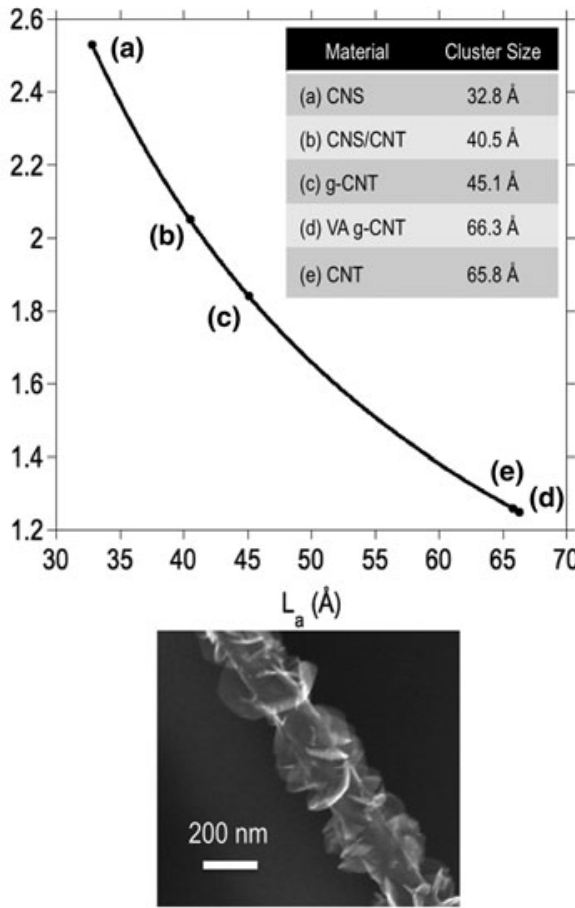

(c)

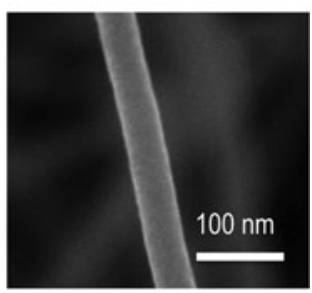

(e)

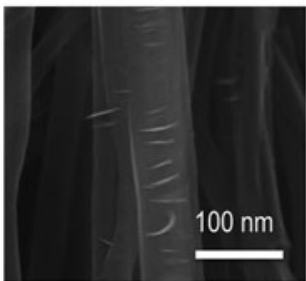

(d)

Figure 2. Nanocrystalline domain size in carbon nanostructures with varying degrees of disorder and graphene edge density. SEMs from (a)-(e) are CNSs, CNS-on-CNTs (plan view, underlying CNT forest not pictured), g-CNTs, VA g-CNTs, and CNTs, and the inset table lists the calculated nanocrystalline domain size for each material.

\section{Results and discussion Relationship between nanocrystalline domain size and nanostructure for high edge density carbon nanostructures}

The classical relationship between the $I_{\mathrm{D}} / I_{\mathrm{G}}$ ratio and nanocrystalline domain size was developed by Tuinstra and Koenig ${ }^{[17]}$ as $L_{\mathrm{a}}=C\left(I_{\mathrm{D}} / I_{\mathrm{G}}\right)^{-1}$, where $L_{\mathrm{a}}$ is the in-plane correlation length and $C=44 \AA$ for an excitation wavelength of $\lambda_{\mathrm{L}}=514.5 \mathrm{~nm}$. Matthews et al. ${ }^{[32]}$ appended this expression to include the wavelength dependence of the constant term, where $C(\lambda)=$ $-126 \AA+0.033 \lambda_{\mathrm{L}}$ for visible wavelengths. Using this expression, the nanocrystalline domain size of each material was calculated (Fig. 2). A clear correlation is observed between the nanostructure observed in the SEM and the nanocrystalline domain size as estimated from the Raman spectra. The CNS nanostructure in Fig. 2(a) contains the highest density and the thinnest nanosheets of all the samples studied, indicating that the crystalline domain size is expected to be the smallest. TEM reported elsewhere ${ }^{[29]}$ has shown that such structures terminate in few-layered graphene, down to two or three layers. The CNS structure in Fig. 2(b) forms at the top of a CNT forest and the thickness of the nanosheets increases dramatically, while the density decreases from the samples in Fig. 2(a), indicating a larger spacing between the edges. As one observes the nanostructure evolution from Figs. 2(c) to 2(e) a decreasing density of graphene edges is observed on the g-CNT sidewalls until no foliates remain and the nanostructure is a standard vertically aligned CNT morphology. Samples shown in Figs. 2(d) and 2(e) are interesting in that both the SEM and the Raman-derived crystalline domain sizes show very minor differences. It is intriguing that the nanocrystalline domain sizes are so similar for these samples, which could imply that FLG may nucleate at the sites of previously existing defects in support of the proposed nucleation and growth model for graphene foliates. ${ }^{[29,33]}$

\section{Interpretation of the nanocrystalline domain size calculation}

The data in Fig. 2 and the discussion above illustrate that the observed edge density follows trends in the Raman-derived crystalline domain size. Although the Tuinstra and Koenig expression originally attempted to link the $D$ peak intensity to phonon confinement, it has been discovered more recently that double resonance is the activation mechanism. ${ }^{[34]}$ Therefore, one may consider $L_{\mathrm{a}}$ as an average interdefect spacing with the expectation that a larger defect density gives rise to a higher $D$ peak intensity, and thus a smaller $L_{\mathrm{a}} \cdot{ }^{\left[{ }^{[5]}\right.}$ Comparing this Raman expression with crystallite size estimates by $\mathrm{x}$-ray 
diffraction, the larger crystallites are given less weight in this case and the above expression underestimates $L_{\mathrm{a}}$ due to the dominant effect of small crystallites. ${ }^{[36-38]}$ Bearing in mind the limitations of the above formulation of crystalline domain size, the trends detailed in this letter are nevertheless tooted in the existence and spatial frequency of defects in the materials. Thus, $L_{\mathrm{a}}$ can be equally referred to as the mean spacing between defects, including the edge defects that are prominent in the materials of the current study.

\section{Relationship between nanocrystalline domain size and specific capacitance}

Data from a previously reported design of experiments (DOE) study of MPECVD growth ${ }^{[29]}$ was used to compare the $I_{\mathrm{D}} / I_{\mathrm{G}}$ ratio and specific capacitance of a variety of carbon nanostructures from CNTs to highly defective amorphous carbon (a-C), thus extending the range of nanostructured carbons beyond those depicted in Fig. 2. Briefly, the DOE process employed statistical methods to populate the process space of growth in the PECVD reactor, analyzing catalyst thickness, pretreatment time, process gas ratio, deposition temperature, and deposition time as factors and presence of CNTs, presence of CNSs, CNT diameter, $I_{\mathrm{D}} / I_{\mathrm{G}}$ ratio, and capacitance as responses.

A monotonically increasing trend was discovered between $I_{\mathrm{D}} / I_{\mathrm{G}}$ and capacitance, which prompted a comparison with $L_{\mathrm{a}}$. As can be seen in Fig. 3, a linear relationship exists between capacitance as a function of nominal surface area and the nanocrystalline domain size calculated from the Raman data.

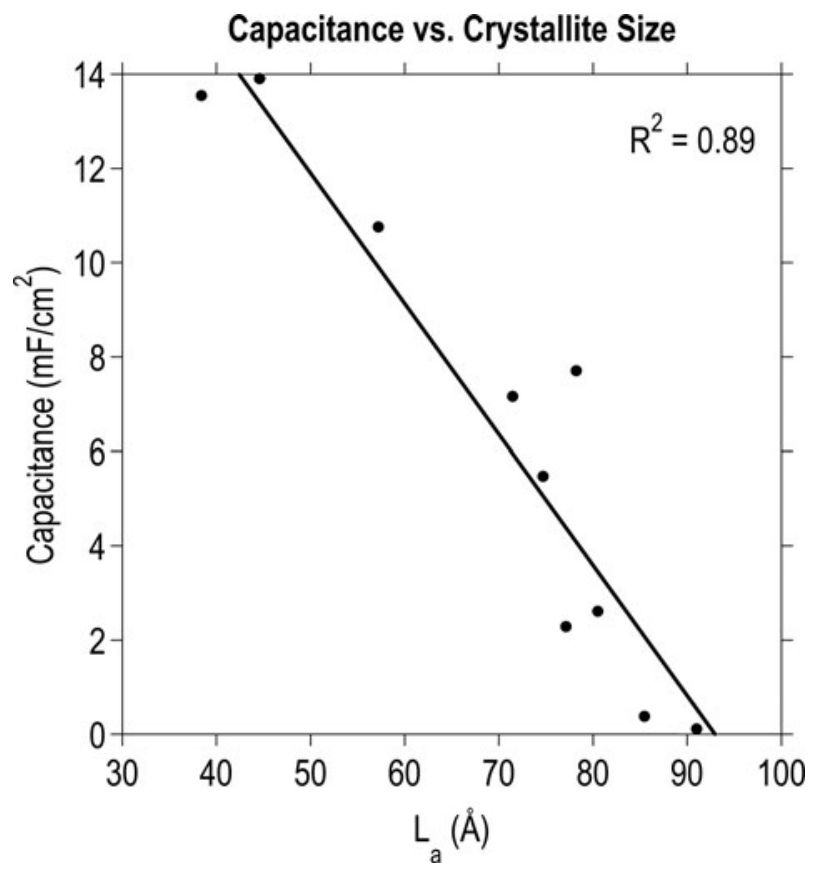

Figure 3. The relationship between nanocrystalline domain size and specific capacitance. A linear fit converges with an $R^{2}$ value of 0.89 for various carbon thin films deposited according to the DOE procedure. ${ }^{[29]}$

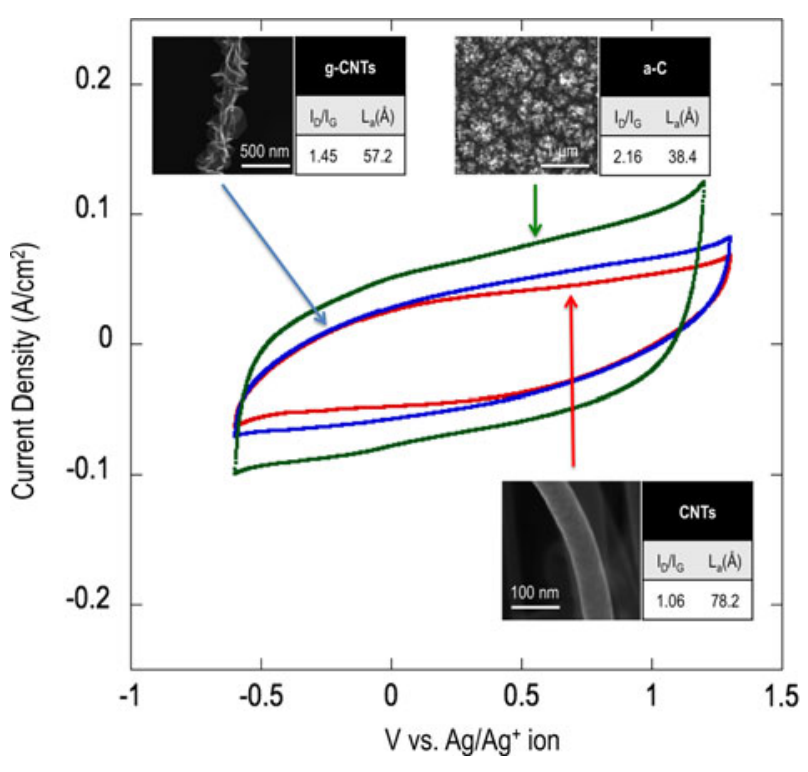

Figure 4. CV curves $(100 \mathrm{mV} / \mathrm{s})$ of structures from the plot in Fig. 3. SEM micrographs are representative images of the nanostructures grown for the capacitance data set, including CNTs, g-CNTs, and a-C.

Fig. 4 illustrates this trend in the form of cyclic voltammetry (CV) curves for three representative films: CNTs, g-CNTs, and a-C. This result is in agreement with reports of Rice and McCreery ${ }^{[23]}$ and others ${ }^{[15,24,39,40]}$ who determined that the specific capacitance of the basal plane was much lower than edge planes in graphitic materials. More recently, it was experimentally demonstrated that the edge of a single graphene sheet possesses a specific capacitance four orders of magnitude higher than the basal plane, a faster electron transfer rate, and stronger electrocatalytic activity. ${ }^{[41]}$ The trend is also consistent with previously reported electrochemical impedance spectroscopy data $^{[28]}$ of g-CNTs, showing an increase of $5.4 \times$ for massnormalized capacitance of g-CNTs compared with CNTs at $1 \mathrm{~Hz}$, and faster charge transfer kinetics in g-CNTs compared with CNTs. ${ }^{[42]}$

By roughly estimating and comparing the surface area of CNTs and CNSs, it is possible to eliminate the possibility that the trend in Fig. 3 arises primarily due to differences in the surface area. CNSs grown by PECVD can be modeled as semi-circular two-dimensional objects with a radius of $\sim 700 \mathrm{~nm}$ and inter-sheet spacing of $\sim 500 \mathrm{~nm}$ based on SEM images. For a substrate of $1 \mathrm{~cm}^{2}$ nominal area, this corresponds to a surface area on the order of $10^{-4} \mathrm{~m}^{2}$. Similarly for CNTs on a $1 \mathrm{~cm}^{2}$ substrate, assuming a CNT diameter of $\sim 50 \mathrm{~nm}$, height $\sim 10 \mu \mathrm{m}$, and density $1.5 \times 10^{10} \mathrm{CNTs} / \mathrm{cm}^{2},{ }^{[31]}$ the surface area is on the order of $10^{-2} \mathrm{~m}^{2}$. Thus, CNTs have approximately two orders of magnitude higher surface area compared with CNSs under these experimental conditions. Since CNSs possess a smaller $L_{\mathrm{a}}$ and higher specific capacitance than CNTs, it can therefore be concluded that defect density, which influences electronic structure and adsorption, more strongly 


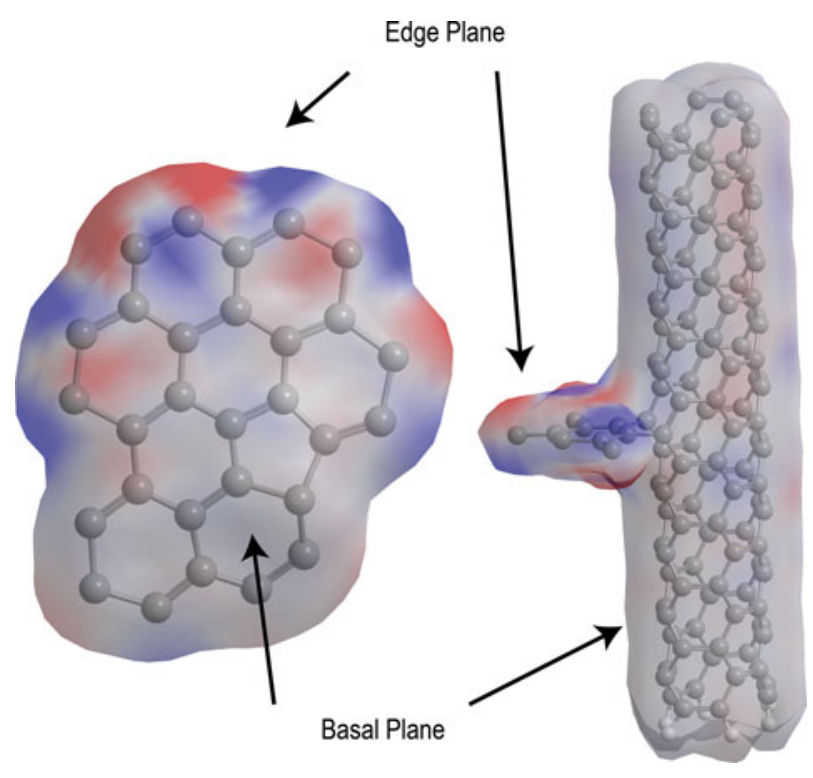

Figure 5. Representation of the molecular orbitals and associated charge densities in a small graphene sheet (left) and a CNT with a graphene foliate (right) calculated using the extended Hückel method. Blue regions are more negatively charged, and red represents positively charged areas. Charge density and associated DOS are higher near the graphene edge planes relative to the basal plane, consistent with the experimental observations.

corresponds to electrochemical capacitance than surface area for these materials.

The density of states (DOS) of pristine graphene, which is 0 at the Fermi level, has been shown to increase with an increase of edge plane defects. ${ }^{[43-45]}$ Furthermore, a high DOS was reported at graphene zig-zag edges. ${ }^{[45]}$ Interestingly, doublelayered graphene is nearly 1.6 times more reactive than singlelayer graphene according to calculations performed by Sharma et al. ${ }^{[46]}$ As the primary class of defects in the hybrid materials presented here is edge plane defects emerging from FLG foliates, an increase in local charge density at these edge plane sites may be primarily responsible for the improvement in double-layer capacitance and charge transfer kinetics. Figure 5 illustrates local differences in charge density for two simplified representative structures: a graphene flake with a pentagon defect and a small foliate covalently bonded to a CNT. Molecular orbitals and charge densities were calculated using the extended Hückel method, and energy was minimized using the MM2 interatomic potential. The edge plane sites display significantly higher charge density compared with the basal planes, in agreement with the literature.

\section{Conclusions}

Carbon nanomaterials with varying defect density were grown via MPECVD and their Raman spectra were analyzed. The $I_{\mathrm{D}} /$ $I_{\mathrm{G}}$ ratio was used to calculate the mean nanocrystalline domain size, which may also be interpreted as the mean distance between defects or in-plane correlation length, ${ }^{[21]}$ including the "foliates" of FLG grown directly on substrates or simultaneously with CNTs. A linear trend emerged between the measured specific capacitance and $L_{\mathrm{a}}$, yielding a novel perspective for engineering carbon nanomaterials for energy storage applications.

\section{Acknowledgments}

We acknowledge Professor Tom O'Haver at the University of Maryland at College Park for development of the Matlab peak fitting script used in this study, and the Shared Materials Instrumentation Facility staff at Duke University for access to the Raman spectrometer and scanning electron microscope. This work was partially supported by grants ECCS1344745 and DMR-1106173 from the National Science Foundation and 1R21NS070033-01A1 from the National Institutes of Health.

\section{References}

1. E. Frackowiak and F. Béguin: Carbon materials for the electrochemical storage of energy in capacitors. Carbon 39, 937 (2001).

2. E. Frackowiak and F. Béguin: Electrochemical storage of energy in carbon nanotubes and nanostructured carbons. Carbon 40, 1775 (2002).

3. J. Gamby, P.L. Taberna, P. Simon, J.F. Fauvarque, and M. Chesneau: Studies and characterisations of various activated carbons used for carbon/carbon supercapacitors. J. Power Sources 101, 109 (2001).

4. D.N. Futaba, K. Hata, T. Yamada, T. Hiraoka, Y. Hayamizu, Y. Kakudate, 0. Tanaike, H. Hatori, M. Yumura, and S. lijima: Shape-engineerable and highly densely packed single-walled carbon nanotubes and their application as super-capacitor electrodes. Nat. Mater. 5, 987 (2006).

5. A. Izadi-Najafabadi, S. Yasuda, K. Kobashi, T. Yamada, D.N. Futaba, H. Hatori, M. Yumura, S. lijima, and K. Hata: Extracting the full potential of single-walled carbon nanotubes as durable supercapacitor electrodes operable at $4 \mathrm{~V}$ with high power and energy density. Adv. Mater. 22, E235 (2010).

6. K.H. An, W.S. Kim, Y.S. Park, Y.C. Choi, S.M. Lee, D.C. Chung, D.J. Bae, S.C. Lim, and Y.H. Lee: Supercapacitors using single-walled carbon nanotube electrodes. Adv. Mater. 13, 497 (2001).

7. E. Frackowiak, K. Metenier, V. Bertagna, and F. Beguin: Supercapacitor electrodes from multiwalled carbon nanotubes. Appl. Phys. Lett. 77, 2421 (2000).

8. M.D. Stoller, S. Park, Y. Zhu, J. An, and R.S. Ruoff: Graphene-based ultracapacitors. Nano Lett. 8, 3498 (2008).

9. Y. Wang, Z. Shi, Y. Huang, Y. Ma, C. Wang, M. Chen, and Y. Chen: Supercapacitor devices based on graphene materials. J. Phys. Chem. C 113, 13103 (2009).

10. Q. Cheng, J. Tang, J. Ma, H. Zhang, N. Shinya, and L.-C. Qin: Graphene and nanostructured $\mathrm{MnO2}$ composite electrodes for supercapacitors. Carbon 49, 2917 (2011).

11. C. Liu, Z. Yu, D. Neff, A. Zhamu, and B.Z. Jang: Graphene-based supercapacitor with an ultrahigh energy density. Nano Lett. 10, 4863 (2010).

12. A. Kuperman and I. Aharon: Battery-ultracapacitor hybrids for pulsed current loads: a review. Renew. Sustain. Energy Rev. 15, 981 (2011).

13. Y. He, W. Chen, X. Li, Z. Zhang, J. Fu, C. Zhao, and E. Xie: Freestanding three-dimensional graphene/MnO2 composite networks as ultralight and flexible supercapacitor electrodes. ACS Nano 7, 174 (2012).

14. J. Zang, S. Ryu, N. Pugno, Q. Wang, Q. Tu, M.J. Buehler, and X. Zhao: Multifunctionality and control of the crumpling and unfolding of largearea graphene. Nat. Mater. 12, 321 (2013).

15. J.R. Miller, R.A. Outlaw, and B.C. Holloway: Graphene double-layer capacitor with ac line-filtering performance. Science 329, 1637 (2010).

16. S.F. Cogan: Neural Stimulation and Recording Electrodes, in Annual Review of Biomedical Engineering (Annual Reviews, Palo Alto, 2008), p. 275. 
17. F. Tuinstra and J.L. Koenig: Raman spectrum of graphite. J. Chem. Phys. 53, 1126 (1970).

18. R.E. Shroder, R.J. Nemanich, and J.T. Glass: Analysis of the composite structures in diamond thin films by Raman spectroscopy. Phys. Rev. B 41, 3738 (1990).

19. R.J. Nemanich and S.A. Solin: First- and second-order Raman scattering from finite-size crystals of graphite. Phys. Rev. B 20, 392 (1979).

20. M.S. Dresselhaus, A. Jorio, M. Hofmann, G. Dresselhaus, and R. Saito: Perspectives on carbon nanotubes and graphene Raman spectroscopy. Nano Lett. 10, 751 (2010).

21. A.C. Ferrari and J. Robertson: Interpretation of Raman spectra of disordered and amorphous carbon. Phys. Rev. B 61, 14095 (2000).

22. T. Kim, S. Lim, K. Kwon, S.-H. Hong, W. Qiao, C.K. Rhee, S.-H. Yoon, and I. Mochida: Electrochemical capacitances of well-defined carbon surfaces. Langmuir 22, 9086 (2006).

23. R.J. Rice and R.L. McCreery: Quantitative relationship between electron transfer rate and surface microstructure of laser-modified graphite electrodes. Anal. Chem. 61, 1637 (1989).

24. R.J. Rice, N.M. Pontikos, and R.L. McCreery: Quantitative correlations of heterogeneous electron-transfer kinetics with surface properties of glassy carbon electrodes. J. Am. Chem. Soc. 112, 4617 (1990).

25. M. Pumera, T. Sasaki, and H. Iwai: Relationship between carbon nanotube structure and electrochemical behavior: heterogeneous electron transfer at electrochemically activated carbon nanotubes. Chem.-Asian J. 3, 2046 (2008).

26. A. Ambrosi, H.L. Poh, L. Wang, Z. Sofer, and M. Pumera: Capacitance of $\mathrm{p}$ - and $\mathrm{n}$-doped graphenes is dominated by structural defects regardless of the dopant type. ChemSusChem 7, 1102 (2014).

27. H. Cui, O. Zhou, and B.R. Stoner: Deposition of aligned bamboo-like carbon nanotubes via microwave plasma enhanced chemical vapor deposition. J. Appl. Phys. 88, 6072 (2000).

28. C.B. Parker, A.S. Raut, B. Brown, B.R. Stoner, and J.T. Glass: Three-dimensional arrays of graphenated carbon nanotubes. J. Mater. Res. 27, 1046 (2012).

29. S.M. Ubnoske, A.S. Raut, B. Brown, C.B. Parker, B.R. Stoner, and J.T. Glass: Perspectives on the growth of high edge density carbon nanostructures: transitions from vertically oriented graphene nanosheets to graphenated carbon nanotubes. J. Phys. Chem. C 118, 16126 (2014).

30. V. Mennella, G. Monaco, L. Colangeli, and E. Bussoletti: Raman spectra of carbon-based materials excited at $1064 \mathrm{~nm}$. Carbon 33, 115 (1995).

31. A.S. Raut, C.B. Parker, and J.T. Glass: A method to obtain a Ragone plot for evaluation of carbon nanotube supercapacitor electrodes. J. Mater. Res. 25, 1500 (2010).

32. M.J. Matthews, M.A. Pimenta, G. Dresselhaus, M.S. Dresselhaus, and M. Endo: Origin of dispersive effects of the Raman $D$ band in carbon materials. Phys. Rev. B 59, R6585 (1999).

33. L. Zeng, D. Lei, W. Wang, J. Liang, Z. Wang, N. Yao, and B. Zhang: Preparation of carbon nanosheets deposited on carbon nanotubes by microwave plasma-enhanced chemical vapor deposition method. Appl. Surf. Sci. 254, 1700 (2008).

34. C. Thomsen and S. Reich: Double resonant Raman scattering in graphite. Phys. Rev. Lett. 85, 5214 (2000).

35. A.C. Ferrari: Raman spectroscopy of graphene and graphite: disorder, electron-phonon coupling, doping and nonadiabatic effects. Solid State Commun. 143, 47 (2007).

36. A. Cuesta, P. Dhamelincourt, J. Laureyns, A. Martinez-Alonso, and J.M. D. Tascon: Comparative performance of $\mathrm{X}$-ray diffraction and Raman microprobe techniques for the study of carbon materials. J. Mater. Chem. 8, 2875 (1998).

37. H. Wilhelm, M. Lelaurain, E. McRae, and B. Humbert: Raman spectroscopic studies on well-defined carbonaceous materials of strong twodimensional character. J. Appl. Phys. 84, 6552 (1998).

38. M. Nakamizo, R. Kammereck, and P.L. Walker Jr.: Laser Raman studies on carbons. Carbon 12, 259 (1974).

39. J.P. Randin and E. Yeager: Differential capacitance study of stressannealed pyrolytic graphite electrodes. J. Electrochem. Soc. 118, 711 (1971).

40. R.L. McCreery: Advanced carbon electrode materials for molecular electrochemistry. Chem. Rev. 108, 2646 (2008).
41. W. Yuan, Y. Zhou, Y. Li, C. Li, H. Peng, J. Zhang, Z. Liu, L. Dai, and G. Shi: The edge- and basal-plane-specific electrochemistry of a single-layer graphene sheet. Sci. Rep. 3, 2248 (2013).

42.P.A. Henry, A.S. Raut, S.M. Ubnoske, C.B. Parker, and J.T. Glass: Enhanced electron transfer kinetics through hybrid graphene-carbon nanotube films. Electrochem. Commun. 48, 103 (2014).

43. N.M.R. Peres, Y. Ling, and T. Shan-Wen: Local density of states and scanning tunneling currents in graphene. New J. Phys. 11, 095007 (2009).

44. R.L. McCreery and M.T. McDermott: Comment on electrochemical kinetics at ordered graphite electrodes. Anal. Chem. 84, 2602 (2012).

45. D.-e Jiang, B.G. Sumpter, and S. Dai: Unique chemical reactivity of a graphene nanoribbon's zigzag edge. J. Chem. Phys. 126, 134701 (2007).

46. R. Sharma, J.H. Baik, C.J. Perera, and M.S. Strano: Anomalously large reactivity of single graphene layers and edges toward electron transfer chemistries. Nano Lett. 10, 398 (2010). 